\title{
Elucidation of the Molecular Mechanism and Exploration of Novel Therapeutics for Spinocerebellar Ataxia Caused by Mutant Protein Kinase $\mathrm{C}_{\gamma}$
}

\author{
Takahiro Seki ${ }^{1, *,}$, Naoko Adachi ${ }^{2}$, Nana Abe-Seki ${ }^{1}$, Takayuki Shimahara ${ }^{1}$, Hideyuki Takahashi ${ }^{2}$, \\ Kazuhiro Yamamoto ${ }^{1}$, Naoaki Saito ${ }^{2}$, and Norio Sakai ${ }^{1}$ \\ ${ }^{1}$ Department of Molecular and Pharmacological Neuroscience, Graduate School of Biomedical Sciences, \\ Hiroshima University, Hiroshima 734-8551, Japan \\ ${ }^{2}$ Laboratory of Molecular Pharmacology, Biosignal Research Center, Kobe University, Kobe 657-8501, Japan
}

Received March 23, 2011; Accepted April 28, 2011

\begin{abstract}
Spinocerebellar ataxia (SCA) is an inherited neurodegenerative disorder that is characterized by cerebellar atrophy and progressive ataxia and is classified into 31 types by the genetic locus. Recently, missense mutations of PRKCG genes that code protein kinase $\mathrm{C} \gamma(\gamma \mathrm{PKC}$ ) have been identified as a causal gene of SCA14. To explore the molecular mechanism of SCA14 pathogenesis, we investigated how mutant $\gamma \mathrm{PKC}$ causes the neurodegeneration of cerebellar Purkinje cells (PCs) by expressing mutant $\gamma \mathrm{PKC}-\mathrm{GFP}$ in cell lines and primary cultured PCs. Mutant $\gamma \mathrm{PKC}$ was susceptible to aggregation in the cytoplasm, which led to an impairment of the ubiquitinproteasome system and apoptosis. Furthermore, mutant $\gamma \mathrm{PKC}$ induced improper dendritic development of cultured PCs in an aggregation-independent manner. Stimulation-induced translocation of mutant $\gamma \mathrm{PKC}$ in $\mathrm{PC}$ dendrites was prominently attenuated by the reduced mobility of oligomerized mutant $\gamma \mathrm{PKC}$, which resulted in attenuated signal transduction and the improper morphology of PC dendrites. These findings suggested that the oligomerization and aggregation of mutant $\gamma \mathrm{PKC}$ caused improper dendritic development and apoptosis of PCs, which led to cerebellar dysfunction and SCA14 pathogenesis. We screened the chemicals that improved these cellular dysfunctions and identified several compounds, including trehalose and Congo red, which could be novel therapeutics for SCA14.
\end{abstract}

Keywords: spinocerebellar ataxia, protein kinase $\mathrm{C} \gamma$, cerebellar Purkinje cell aggregation, apoptosis

\section{Introduction}

The autosomal dominant spinocerebellar ataxias (SCAs) are a heterogeneous group of neurological disorders that are clinically characterized by the progressive ataxia of gait and limbs, cerebellar dysarthria, and abnormal eye movements. SCAs are classified into at least 31 types (SCA $1-31$ ) by the different genetic loci of the

*Corresponding author. tseki@hiroshima-u.ac.jp Published online in J-STAGE on June 10, 2011 (in advance) doi: 10.1254/jphs.11R04CP causal gene $(1,2)$. The involved genes and the responsible mutations have been identified in more than half of the SCAs. Among these, CAG trinucleotide repeat expansions are most frequently found in 7 types of SCAs (SCA $1,2,3,6,7,12$, and 17) and the SCA-related disorder dentatorubral pallidoluysian atrophy (DRPLA) (1). Diseases caused by such expansions, including Huntington disease and spinal and bulbar muscular atrophy (SBMA), are called polyglutamine diseases (3). The aggregation of mutant proteins that have an abnormally elongated polyglutamine tract is considered to be the molecular basis of neuronal degeneration in polyglutamine diseases (3).

${ }^{\S}$ This review article is written on the occasion of the author (Takahiro Seki) receiving the 26th Award for Encouragement of Young Investigators from The Japanese Pharmacological Society in 2011 and on the basis of the targeted work. 
Thereafter, missense mutations and deletions have been found in other SCAs (SCA 5, 11, 13, 14, 15, and 27) (1). Although various causal genes have been identified, the molecular pathogenesis of SCAs has not been elucidated, and there are no convincing therapeutics for SCA treatment.

In 2003, Chen et al. demonstrated that SCA14 is caused by missense mutations in the $P R K C G$ gene that encodes protein kinase $\mathrm{C} \gamma(\gamma \mathrm{PKC})$ (4). To date, 24 mutations ( 22 missense mutations and 2 deletions) have been identified in different SCA14 families (Fig. 1) $(5,6)$. $\mathrm{PKC}$ is a family of serine/threonine kinases that play important roles in various cellular functions by participating in diverse signal transduction pathways (7). The subtype $\gamma \mathrm{PKC}$, specifically present in the central nervous system, is especially abundant in cerebellar Purkinje cells (8). Although $\gamma$ PKC-knockout mice show mildly impaired motor coordination (9), this ataxic symptom is milder than the impairments that are found in SCA14 patients. Moreover, SCA14 is inherited in an autosomal dominant fashion, which raises the possibility that a toxic gain of function of mutant $\gamma \mathrm{PKC}$, rather than a loss of function, underlies the pathogenesis of SCA14.

We have previously established live imaging studies using green fluorescent protein (GFP)-tagged PKC (PKC-GFP) and demonstrated that PKCs are translocated to several cellular organelles in a subtype- and stimulation-specific manner when PKCs are activated by various stimulations $(10,11)$. Thereafter, PKCs recognize and phosphorylate their substrates in the targeted subcellular regions and cause the subsequent cellular responses (i.e., PKC targeting). This PKC targeting is the molecular basis that underlies the multiplicity of PKC-mediated functions. Using transgenic mice that overexpress $\gamma \mathrm{PKC}$ GFP, we have reported that the translocation of $\gamma \mathrm{PKC}$ GFP, which was induced by the electrical stimulation of parallel fibers, propagates along the dendritic shaft of cerebellar Purkinje cells (12), which indicates that PKC targeting is a prerequisite for various $\mathrm{PKC}$-involved neuronal functions in Purkinje cells. To elucidate how mutant $\gamma \mathrm{PKCs}$ induce neuronal degeneration and the pathology of SCA14, we expressed mutant $\gamma$ PKC-GFP in cultured cell lines and primary cultured PCs and investigated the molecular properties of mutant $\gamma \mathrm{PKC}$, including cellular localization, kinase activity, PKC targeting, and the deleterious effects on cells, and compared these responses with the wild type.

\section{Aggregation, oligomerization, and the apoptosis induction of mutant $\gamma \mathrm{PKC}$}

In 2005, we first demonstrated the molecular properties of 8 SCA1 14 mutant $\gamma$ PKC-GFPs expressed in Chinese hamster ovary (CHO) cells (13). Most mutant $\gamma \mathrm{PKC}$ GFPs tended to form cytoplasmic aggregates, but wildtype (WT) $\gamma$ PKC-GFP was expressed uniformly in the cytoplasm (Fig. 2A). Aggregates of mutant $\gamma$ PKC-GFP were classified into two types, massive aggregation near the nucleus and a diffuse dot-like aggregation in the cytoplasm (Fig. 2A). Long-term time-lapse imaging revealed that the dot-like aggregation of mutant $\gamma$ PKC-GFP was first observed in the cytoplasm and accumulated in perinuclear regions, which led to the formation of massive aggregates and apoptotic cell death (14). In addition, mutant $\gamma$ PKC-GFP tended to be insoluble in lysis buffer that contained 1\% Triton X-100. Most mutant $\gamma$ PKCGFPs were strongly detected in the Triton-insoluble (I) fraction by immunoblotting with an anti-GFP antibody, but WT $\gamma$ PKC-GFP was similarly detected both in Tritonsoluble (S) and I fractions (Fig. 2B). The decrease in the solubility of mutant $\gamma \mathrm{PKC}$ is probably due to its aggregate-prone property because the solubility of mutant $\gamma$ PKC-GFP is negatively paralleled with its tendency for aggregation (13). The cytotoxicity of mutant $\gamma \mathrm{PKC}-\mathrm{GFP}$

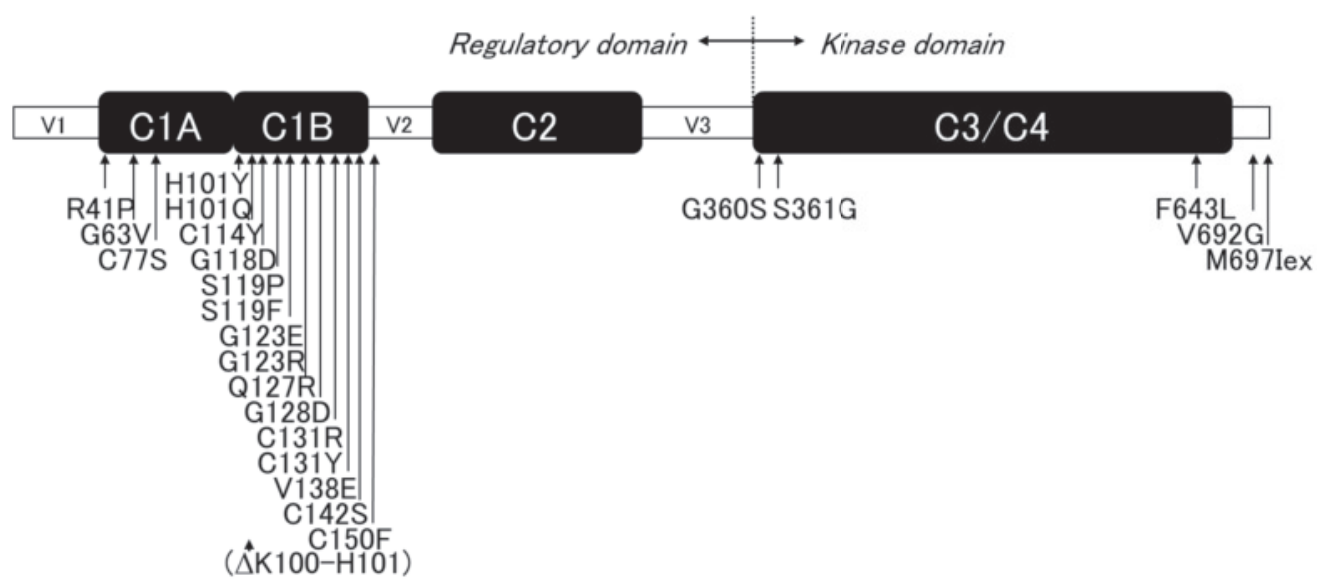

Fig. 1. Schematic illustrations of $\gamma \mathrm{PKC}$ protein structure and the mutations found in SCA14 families. Twenty-two missense mutations and two deletion mutations ( $\triangle \mathrm{K} 100-\mathrm{H} 101$ and M697Iex) are listed in the box below the diagram of $\gamma \mathrm{PKC}$ protein structure. M697Iex indicates a missense change (M697I) and a C-terminal 13-amino-acid extension by a deletion that includes a stop codon (24). 
was quantitatively assessed using flow cytometry with 7-amino-actinomycin D (7-AAD), a fluorescent dye that selectively stains dead cells. Mutant $\gamma$ PKC significantly increased cell death, especially in highly expressing cells (Fig. 2C). In addition to the 8 mutants reported in 2005 , we analyzed aggregation, solubility to Triton, and the cytotoxicity of mutant $\gamma$ PKC-GFP in 19 of 24 reported mutations from SCA14 families (Table 1). Except for 2 mutants (S119F and V692G), most mutant $\gamma$ PKC-GFP formed aggregates and were insoluble in Triton. Moreover, mutant $\gamma$ PKC-GFP also tended to form aggregates and induce apoptosis in primary cultured cerebellar Purkinje cells (PCs) (15). These findings suggest that the aggregate-prone property of mutant $\gamma \mathrm{PKC}$ would be involved in the pathogenesis of SCA14. Similar aggregate formation of mutant or misfolded proteins is frequently

A

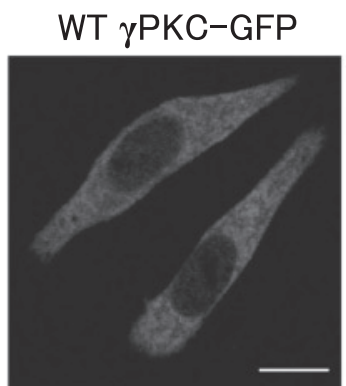

No aggregation

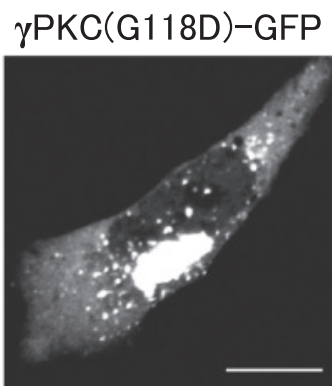

Massive aggregation

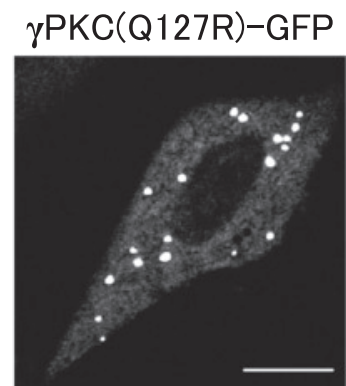

Dot-like aggregation

B

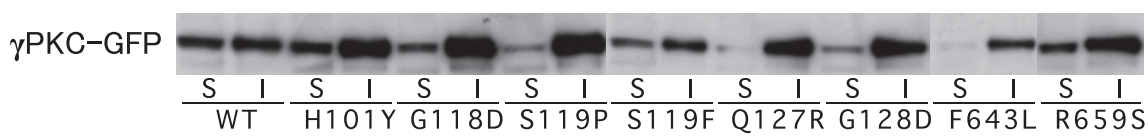

C
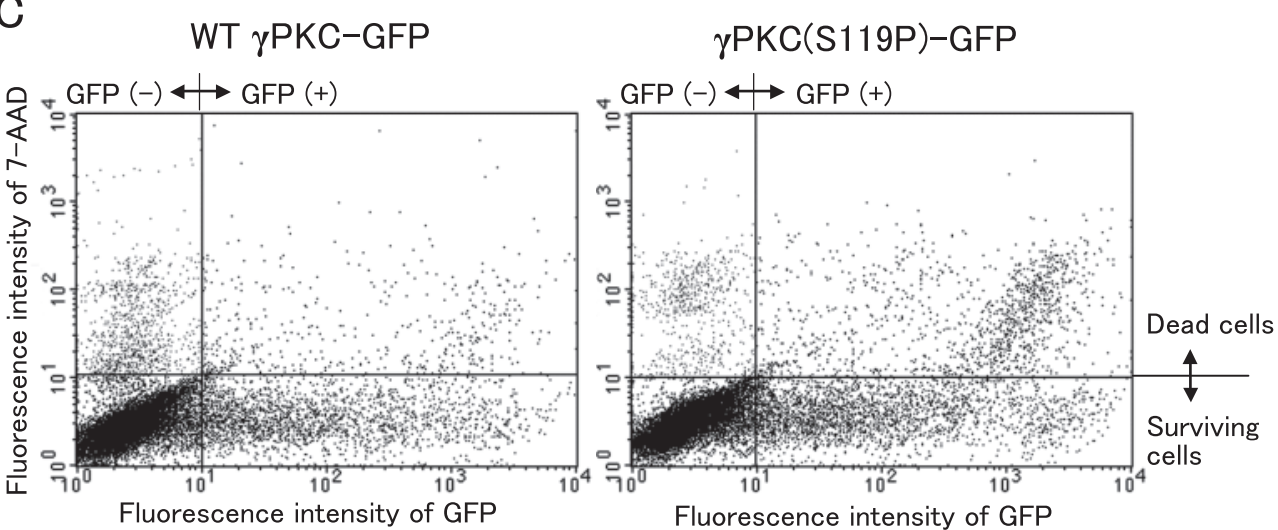

Fig. 2. Aggregation and cytotoxicity of mutant $\gamma \mathrm{PKC}-\mathrm{GFP}$ expressed in CHO cells. A: Representative GFP fluorescent images of wild-type (WT) or mutant $\gamma$ PKC-GFP expression in CHO cells. WT $\gamma$ PKC-GFP was uniformly expressed in the cytoplasm (left). Massive aggregation near the nucleus (center) in the cytoplasm were frequently observed in $\mathrm{CHO}$ cells expressing mutant G118D $\gamma$ PKC-GFP, and dot-like aggregations (right) were observed in the cells expressing mutant Q127R $\gamma$ PKC-GFP. Bar $=10$ $\mu \mathrm{m}$. B: Immunoblotting analysis of Triton-soluble (S) and Triton-insoluble (I) fractions, which were prepared from CHO cells expressing wild-type (WT) and 8 mutant $\gamma$ PKC-GFPs, was performed using an anti-GFP antibody. Two days after transfection, $\mathrm{CHO}$ cells expressing $\gamma \mathrm{PKC}$-GFP were harvested and separated into $\mathrm{S}$ and I fractions by the solubility to lysis buffer containing $1 \%$ Triton X-100. Mutant $\gamma$ PKC-GFP became insoluble to $1 \%$ Triton due to its aggregation. Data shown are representative of four experiments. C: Representative GFP and 7-amino-actinomycin D (7-AAD) dot plots of CHO cells expressing WT (left) and S119P (right) mutant $\gamma$ PKC-GFPs analyzed by flow cytometry. Cells in the right upper and lower quadrants [GFP $(+)$ ] were considered to express $\gamma$ PKC-GFP. Cells in the upper right and left quadrants were considered 7-AAD-positive dead cells. Dead cells in $\gamma$ PKC-GFP-expressing cells were obviously increased in cells that expressed mutant $\gamma$ PKC-GFP. This figure was reproduced and rearranged from the original publication (13) with permission from the American Society for Biochemistry and Molecular Biology. 
Table 1. Aggregation, solubility to Triton, and the cytotoxicity of various SCA14 mutant $\gamma \mathrm{PKC}-\mathrm{GFPs}$ in CHO cells

\begin{tabular}{|c|c|c|c|}
\hline & $\begin{array}{c}\text { Aggregation } \\
(\% \text { of } \gamma \mathrm{PKC}-\mathrm{GFP}- \\
\text { expressing cells })\end{array}$ & $\begin{array}{l}\text { Solubility to Triton } \\
\text { (\% of total } \gamma \text { PKC-GFP) }\end{array}$ & $\begin{array}{c}\text { Cytotoxicity } \\
\text { (\% of } \gamma \mathrm{PKC}-\mathrm{GFP}- \\
\text { expressing cells) }\end{array}$ \\
\hline WT & $23.8 \pm 3.4$ & $32.0 \pm 4.8$ & $20.6 \pm 0.4$ \\
\hline $\mathrm{R} 41 \mathrm{P}$ & $55.1 \pm 6.2 *$ & $13.3 \pm 2.0$ & $27.7 \pm 1.3^{*}$ \\
\hline$\Delta \mathrm{K} 100-\mathrm{H} 101$ & $54.5 \pm 2.3^{*}$ & $8.7 \pm 0.7^{*}$ & $28.0 \pm 1.3^{*}$ \\
\hline H101Q & $66.6 \pm 8.2 *$ & $9.8 \pm 2.0^{*}$ & $26.7 \pm 1.4^{*}$ \\
\hline H101Y & $59.5 \pm 3.6^{*}$ & $12.3 \pm 3.2^{*}$ & $29.4 \pm 3.6^{*}$ \\
\hline $\mathrm{C} 114 \mathrm{Y}$ & $53.1 \pm 1.8^{*}$ & $6.9 \pm 1.2^{*}$ & $32.7 \pm 2.0^{*}$ \\
\hline G118D & $41.4 \pm 5.6^{*}$ & $17.3 \pm 5.7$ & $22.3 \pm 1.3$ \\
\hline S119P & $60.1 \pm 6.4^{*}$ & $8.8 \pm 2.6^{*}$ & $31.5 \pm 2.5^{*}$ \\
\hline S119F & $35.0 \pm 5.2$ & $47.8 \pm 17.0$ & $23.5 \pm 1.5^{*}$ \\
\hline G123E & $49.7 \pm 4.7^{*}$ & $6.9 \pm 3.0^{*}$ & $27.5 \pm 0.8^{*}$ \\
\hline G123R & $57.1 \pm 2.5^{*}$ & $8.9 \pm 4.0^{*}$ & $22.5 \pm 2.1$ \\
\hline Q127R & $49.8 \pm 2.8^{*}$ & $6.7 \pm 1.7^{*}$ & $25.5 \pm 1.8^{*}$ \\
\hline G128D & $56.9 \pm 11.9 *$ & $5.7 \pm 1.0^{*}$ & $26.5 \pm 2.5^{*}$ \\
\hline C131R & $69.8 \pm 2.8^{*}$ & $13.4 \pm 3.4^{*}$ & $33.3 \pm 0.6^{*}$ \\
\hline V138E & $56.5 \pm 2.1^{*}$ & $14.7 \pm 3.3$ & $30.9 \pm 1.3^{*}$ \\
\hline $\mathrm{C} 150 \mathrm{~F}$ & $49.6 \pm 1.5^{*}$ & $12.8 \pm 1.5$ & $24.5 \pm 2.3^{*}$ \\
\hline G360S & $45.7 \pm 3.5^{*}$ & $7.8 \pm 3.0^{*}$ & $27.3 \pm 0.8^{*}$ \\
\hline S361G & $36.6 \pm 2.7^{*}$ & $11.9 \pm 1.1$ & $29.0 \pm 4.5^{*}$ \\
\hline F643L & $45.6 \pm 4.3^{*}$ & $7.4 \pm 1.7^{*}$ & $25.5 \pm 2.3^{*}$ \\
\hline V692G & $28.9 \pm 2.9$ & $17.1 \pm 3.4$ & $25.0 \pm 1.8^{*}$ \\
\hline
\end{tabular}

The analyses shown in Fig. 2 were conducted in 19 mutant $\gamma$ PKC-GFPs. Aggregation was quantified as the percentage of cells with aggregates in cells expressing $\gamma \mathrm{PKC}$-GFP. Solubility to Triton was quantified as the percentage of the amount of $\gamma \mathrm{PKC}$-GFP in the $\mathrm{S}$ fraction in the total amount of $\gamma \mathrm{PKC}-\mathrm{GFP}(\mathrm{S}+\mathrm{I}$ fractions). Cytotoxicity was quantified as the percentage of dead cells in total $\gamma \mathrm{PKC}-\mathrm{GFP}-$ expressing cells, as analyzed by flow cytometry. An asterisk represents a significant change $(P<0.05)$ from WT $\gamma \mathrm{PKC}$-GFP, as analyzed by the unpaired $t$-test.

observed in other neurodegenerative disorders, including Parkinson's disease, Alzheimer's disease, amyotrophic lateral sclerosis, and polyglutamine disease (16). These findings indicated that aggregation is a common component of the pathogenesis of neurodegenerative diseases like SCA14.

In spite of the frequent observation of aggregation, how the aggregation of mutant proteins causes cell death and neurodegeneration has not been fully elucidated. Because these aggregates are often ubiquitin-positive and sequesters components of the proteasome, the ubiquitin-proteasome system (UPS) is involved in the pathogenesis of neurodegenerative diseases (17). UPS is one of the major proteolytic pathways in mammalian cells, and it is involved in the degradation of cytosolic short-lived proteins, including misfolded proteins (18). The age-related decline of UPS function results in an increased accumulation and aggregation of misfolded proteins. Therefore, UPS dysfunction is one of the etiologies of neurodegenerative diseases (19). Furthermore, several studies have shown that protein aggregation impairs UPS function (19), which leads to the further accumulation of misfolded proteins and to the onset of neurodegenerative diseases. In the case of the SCA14 mutant $\gamma \mathrm{PKC}$, we demonstrated that it is ubiquitinated (14) and degraded via UPS (20). Moreover, aggregates of mutant $\gamma \mathrm{PKC}$ contain proteasome subunits and impair protein degradation via UPS $(14,15)$, which results in the induction of endoplasmic reticulum (ER) stress [e.g., elevation of nuclear CHOP (CCAAT/enhancer binding protein $(\mathrm{C} / \mathrm{EBP})$ homologous protein) and phosphorylated PERK (PKR-like ER kinase)] and apoptotic cell death (14). However, two mutant (S119F and V692G) $\gamma$ PKC-GFPs did not have aggregate-prone properties but induced cell death (Table 1). This result suggests that there are mechanisms other than aggregation that induce 
neurodegeneration.

Increasing evidence has been reported that oligomers, rather than aggregates, of misfolded proteins are toxic to neurons and that the aggregates are formed as a result of cellular protective mechanisms against toxic oligomers (21). We have confirmed that mutant (S119P and G128D) $\gamma \mathrm{PKC}$ form oligomers using blue-native PAGE (15). However, the apoptosis that is triggered by mutant $\gamma$ PKC-GFP was observed only in PCs with aggregates of mutant $\gamma \mathrm{PKC}-\mathrm{GFP}(15)$, which strongly suggests that the aggregation of mutant $\gamma \mathrm{PKC}$ is toxic to PCs. However, because long-term time-lapse observation revealed that there were surviving PCs in large aggregates (15), the aggregation of mutant $\gamma \mathrm{PKC}$ might be important, but not essential, to the induction of apoptosis in PCs.

\section{Kinase activity, targeting, and substrate phos- phorylation of mutant $\gamma$ PKC}

$\gamma \mathrm{PKC}$ is a member of the classical PKCs, which are activated by diacylglycerol (DG) and $\mathrm{Ca}^{2+}$ in the presence of phosphatidylserine (7). $\gamma \mathrm{PKC}$ has $\mathrm{C} 1$ and $\mathrm{C} 2$ domains, which bind $\mathrm{DG}$ and $\mathrm{Ca}^{2+}$, respectively, in its regulatory domain. Of the 24 mutations of $\gamma \mathrm{PKC}$ found in SCA14 families, 19 mutations are located around the $\mathrm{C} 1$ domain (Fig. 1), which binds to DG and phorbol ester, a well-known PKC activator. Therefore, it is possible that these missense mutations influence the kinase activity and targeting of $\gamma \mathrm{PKC}$. In vitro kinase assays have revealed that most mutant $\gamma \mathrm{PKCs}$ have higher basal kinase activities (i.e., without a PKC activator), which are not increased in the presence of PKC activators (22). Similar elevations of basal kinase activity have also been reported from other groups $(23,24)$. This property was observed in SCA14 mutants whose mutations existed in domains other than the $\mathrm{C} 1$ domain. Why does mutant $\gamma$ PKC have higher basal kinase activity? PKC takes a "closed" conformation in which its kinase domain is masked by a pseudosubstrate region in its regulatory domain in the basal condition. The binding of a PKC activator induces an "open" conformation by the release of the pseudosubstrate region from the kinase domain, which enables other PKC substrates to access its kinase domain (25). The elevation of the basal kinase activity of mutant $\gamma \mathrm{PKC}$ is probably due to its conformational change from the "closed" to "open" state, which has been confirmed by Förster resonance energy transfer (FRET) analysis (26). This conformational change might induce the aberrant phosphorylation of cytosolic substrates that are not phosphorylated by WT $\gamma \mathrm{PKC}$. Asai et al. have recently revealed that mutant $\gamma \mathrm{PKC}$ induces the aberrant phosphorylation and dysregulation of aprataxin (APTX) (24), which is a DNA repair protein and is causative for autosomal recessive ataxia (27). There might be cytoplasmic proteins other than APTX that are aberrantly phosphorylated and dysregulated by mutant $\gamma \mathrm{PKC}$ as causal proteins of SCA14. Furthermore, because protein aggregation is caused by protein misfolding, the aberrant conformation in SCA14 mutations should also cause the aggregation of mutant $\gamma \mathrm{PKC}$ and the elevation of basal kinase activity, both of which might induce the neurodegeneration of PCs. Asai et al. also revealed that the inhibition of the kinase activity of mutant $\gamma \mathrm{PKC}$ alleviated its aggregation. This finding indicates that the aggregateprone property of mutant $\gamma \mathrm{PKC}$ might be related to its kinase activity.

As to $\gamma \mathrm{PKC}$ targeting, mutant $\gamma \mathrm{PKC}$-GFP transiently translocates from the cytoplasm to the plasma membrane in response to receptor activation (e.g., P2Y receptor activation by ATP) in CHO cells without aggregates, similar to the WT. However, dot-like aggregation is rapidly induced after reversion to the cytoplasm in several mutant $\gamma$ PKC-GFPs (13), probably due to the conformational change induced by PKC activation. Although WT and mutant $\gamma$ PKCs are translocated to the plasma membrane by various stimulations, the rate and duration of translocation are significantly different between WT and mutant $\gamma$ PKCs. Verbeek et al. have demonstrated that mutant $\gamma \mathrm{PKC}$ rapidly translocates to the plasma membrane in response to calcium ionophore and phorbol ester $(23,26)$. We have revealed previously that mutant $\gamma \mathrm{PKC}$, especially the $\mathrm{C} 1$ domain mutant, shows prolonged membrane localization after translocation in response to receptor activation (22). This effect was due to sustained $\mathrm{Ca}^{2+}$ influx from TRPC 3 , which is phosphorylated and inactivated by WT $\gamma \mathrm{PKC}$ but not by mutant $\gamma \mathrm{PKC}$. This and findings by another group (26) indicate that mutant $\gamma \mathrm{PKC}$ does not fully phosphorylate substrates at the plasma membrane in spite of prolonged membrane localization. Why does mutant $\gamma \mathrm{PKC}$ fail to phosphorylate membrane substrates, like TRPC 3 , despite its high basal kinase activity? Single-molecule observation of $\gamma$ PKC-GFP using total internal reflection fluorescence (TIRF) microscopy revealed that the membrane residence time of $\mathrm{C} 1$ domain mutant $\gamma \mathrm{PKC}$ was significantly shorter than WT in the presence of a PKC activator (22). These results indicate that the $\mathrm{C} 1$ domain mutant $\gamma \mathrm{PKC}$ has a reduced ability to bind DG and phorbol ester and cannot fully access membrane substrate, although it shows apparent prolonged membrane localization. Altogether, mutant $\gamma \mathrm{PKC}$ has a higher kinase activity in the cytoplasm and a reduced activity when it is activated at the plasma membrane. This aberrant regulation of kinase activity could contribute to the pathogenesis of SCA14. 


\section{Aberrant morphology of PC dendrites triggered by mutant $\gamma$ PKC}

In addition to aggregation and apoptosis induction, mutant $\gamma \mathrm{PKC}$ in primary cultured $\mathrm{PCs}$ induces an improper development of PC dendrites (15). Mutant $\gamma \mathrm{PKC}$ reduces dendritic area, dendritic length, and dendritic branches in an aggregate-independent manner (Fig. 3, upper panels). Furthermore, mutant $\gamma \mathrm{PKC}$ decreases the density of dendritic spines with parallel fibers in which GluR $\delta 2$ is specifically localized (15). A previous report demonstrated that $\gamma \mathrm{PKC}$ negatively regulates the dendritic development of PCs (28). It is possible that the higher basal activity of mutant $\gamma \mathrm{PKC}$ triggers the improper dendritic development. However, because mutant $\gamma \mathrm{PKC}$ has a reduced activity at the plasma membrane, the normal signal pathway that is mediated by $\gamma \mathrm{PKC}$ would be disturbed by an SCA14 mutation. Indeed, mutant $\gamma \mathrm{PKC}$ in $\mathrm{PC}$ dendrites has remarkably reduced mobility, probably because of oligomer formation, and shows attenuated translocation to the plasma membrane in response to high $\mathrm{K}^{+}$-induced depolarization (15). Because the translocation of $\gamma \mathrm{PKC}$ propagates along PC dendrites after parallel fiber stimulation in $\gamma \mathrm{PKC}$-GFP transgenic mice (12), it plays an important role in the intracellular signal transduction of PC dendrites. Soluble oligomers of mutant $\gamma \mathrm{PKC}$ would disrupt this signal transduction by attenuating translocation and reducing the phosphorylation of membrane substrates, which would result in reduced dendritic development and spine density.

\section{Exploration of novel therapeutics against dysfunc- tions triggered by mutant $\gamma \mathrm{PKC}$}

Next, we attempted to explore small compounds that are available as novel therapeutics for SCA14. If apoptosis and aberrant dendritic morphology are triggered by the aggregation and oligomerization of mutant $\gamma \mathrm{PKC}$, compounds that inhibit aggregation and oligomerization should alleviate these dysfunctions. We screened several chemicals against aggregation and the cytotoxicity of mutant $\gamma$ PKC-GFP that was expressed in cultured cell lines and identified two aggregate inhibitory drugs that were effective against the dysfunctions triggered by mutant $\gamma$ PKC.

Trehalose is a natural disaccharide of two glucose molecules that protects proteins from denaturation and aggregation as a chemical chaperone (29). Trehalose in-

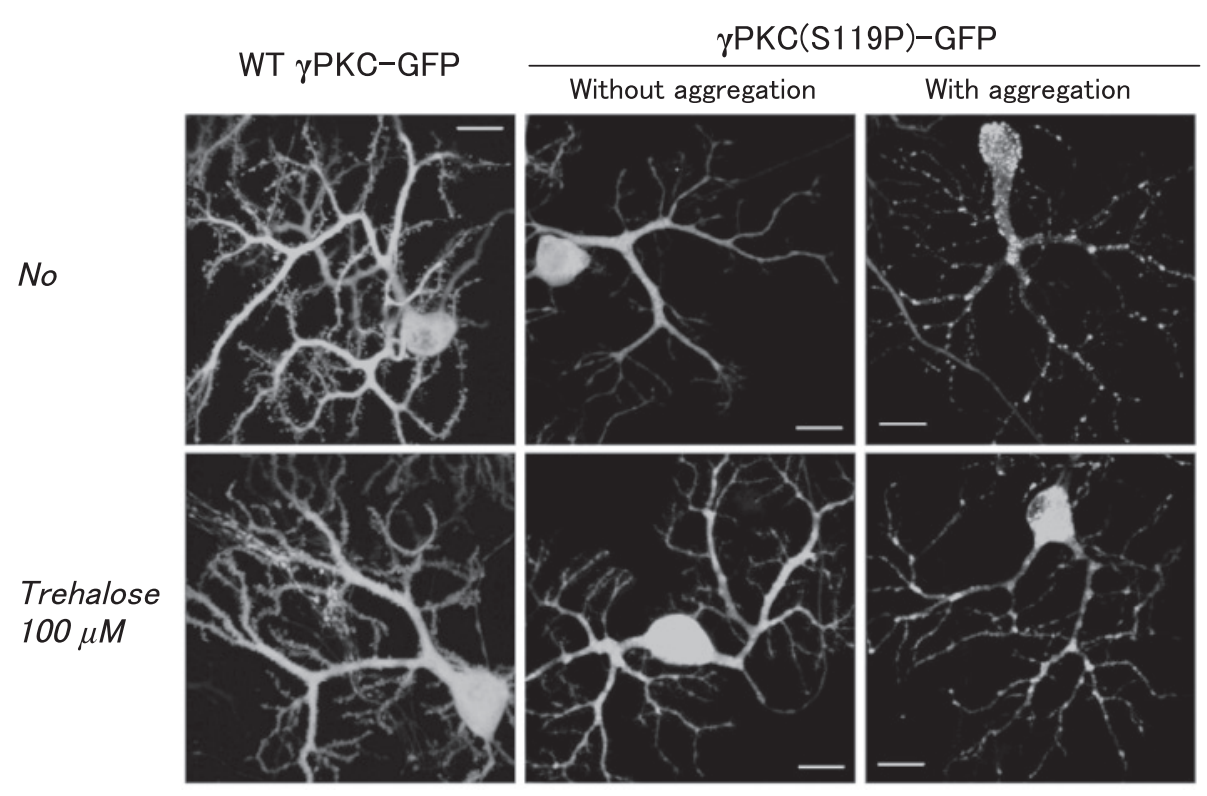

Fig. 3. Aggregation and improper dendritic development in PCs expressing mutant $\gamma \mathrm{PKC}$-GFP. Representative images of GFP fluorescence in living PCs expressing WT $\gamma$ PKC-GFP (left) and S119P mutant $\gamma$ PKC-GFP without (center) and with (right) aggregates. Upper and lower images represent PCs treated without or with $100 \mu \mathrm{M}$ trehalose, respectively. Images were projected from Z-stack images obtained using a confocal laser microscope. These cells were infected with adenoviral vectors on DIV14 and were observed on DIV28. Trehalose was applied at the same time as adenoviral infection and the treatment continued for 14 days. PCs expressing WT $\gamma$ PKC-GFP had highly branched dendrites, but PCs expressing mutant $\gamma$ PKC-GFP had leaner dendrites with reduced branches in an aggregate-independent manner. Trehalose improved the dendritic development in PCs without aggregates of mutant $\gamma$ PKC-GFPs, but not PCs with aggregates. Bar $=20 \mu \mathrm{m}$. This figure was reproduced from the original publication (31) with permission from the American Society for Biochemistry and Molecular Biology. 
hibits the aggregation of mutant proteins and the pathology of several mouse models of neurodegenerative diseases (30). We have recently demonstrated that trehalose inhibits aggregation and apoptosis induction of mutant $\gamma \mathrm{PKC}$ in SH-SY5Y cells and primary cultured PCs (31). Because the inhibition of apoptosis was not observed in PCs with preformed aggregates before trehalose treatment, this effect was mediated by the aggregation inhibitory property of trehalose. Furthermore, trehalose significantly improved dendritic retraction in PCs without aggregates of mutant $\gamma \mathrm{PKC}$, but not in PCs with aggregates (Fig. 3, lower panels). In PCs without aggregates, trehalose alleviated the reduced mobility and the attenuated translocation of mutant $\gamma \mathrm{PKC}$ (31), which suggested that trehalose ameliorated the dendritic development of PCs through the inhibition of oligomerization of mutant $\gamma$ PKC. Because trehalose is effective by oral administration in a mouse model of Huntington's disease (30), it could reach the brain parenchyma through the bloodbrain barrier (BBB) and be available as a novel therapeutic for the treatment of SCA14.

We also examined the effects of Congo red (32), which is widely used as an amyloid dye and has the ability to inhibit oligomerization and fibrillization with an amyloid structure (33). Similar to trehalose, Congo red inhibited aggregation and oligomerization of mutant $\gamma \mathrm{PKC}$, thereby preventing the induction of apoptosis and alleviating aberrant morphology of PCs. This finding also supports the idea that mutant $\gamma \mathrm{PKC}$ tends to form oligomers and aggregates with amyloid structures, which is similar to other neurodegenerative diseases (16), because its oli- gomerization and aggregation was inhibited by Congo red. Although Congo red could be an effective treatment for dysfunctions that are triggered by mutant $\gamma \mathrm{PKC}$, it is not an ideal compound for the treatment of SCA14 because of its low permeability to the BBB, the carcinogenic activity of its metabolites, and its toxicity (33). We confirmed that a higher concentration of Congo red induced an aberrant morphology of PC dendrites (32). Because there are several derivatives of Congo red that have an improved ability to cross the BBB (33), these derivatives may be more suitable for SCA14 treatment than Congo red.

\section{Conclusion}

Altogether, the mutant $\gamma \mathrm{PKC}$ that is found in SCA14 is susceptible to oligomerization and aggregation, which leads to an aberrant morphology of PC dendrites and the apoptosis of PCs, respectively, and to cerebellar dysfunction and the pathogenesis of SCA14 (Fig. 4). Because mutant $\gamma \mathrm{PKC}$ is more rapidly degraded than WT (20), an age-related decline of the UPS might induce the accumulation of mutant $\gamma \mathrm{PKC}$, which triggers its oligomerization and aggregation and leads to a late-onset pathogenesis of SCA14. Although the altered kinase activity of mutant $\gamma \mathrm{PKC}$ might participate in the aberrant dendritic morphology and apoptosis of PCs, its aggregate-prone properties would be more important in these dysfunctions because the aggregate inhibitory compounds ameliorated these dysfunctions. However, it is possible that trehalose and Congo red improved the kinase activity of mutant

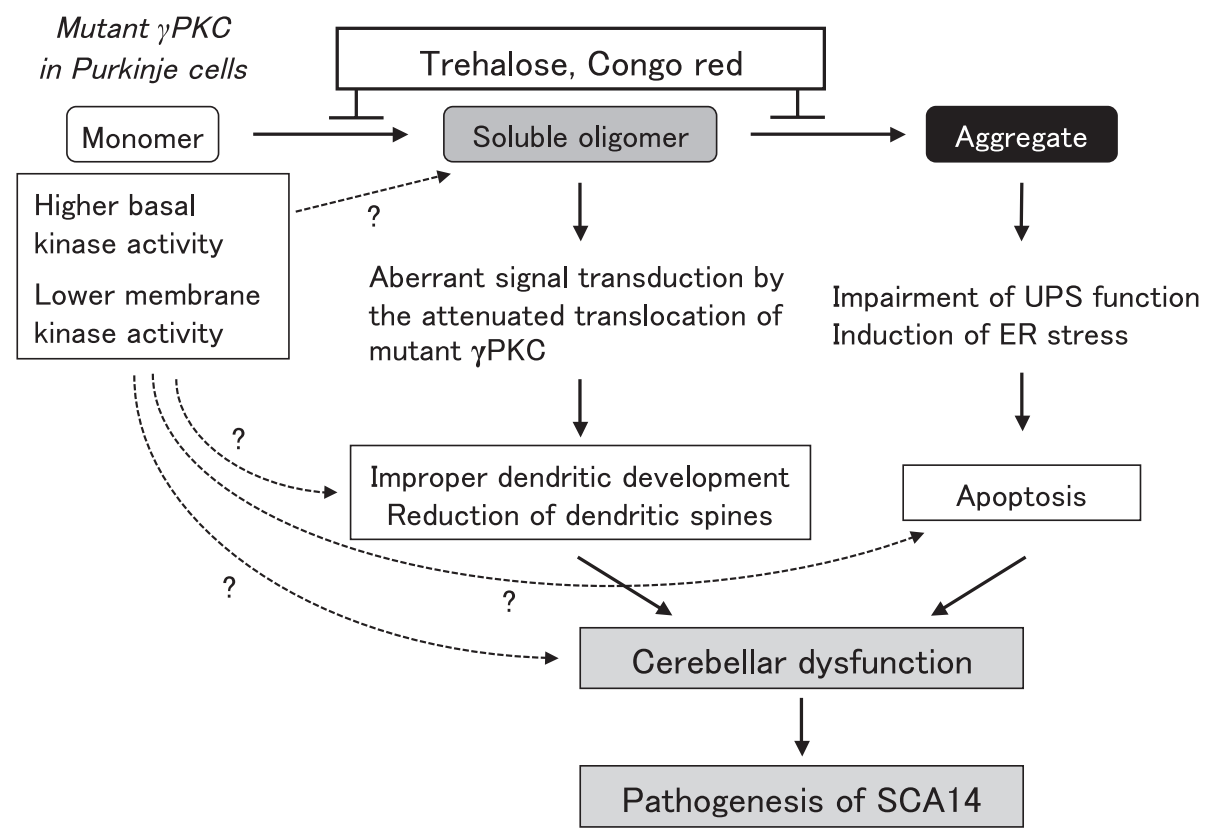

Fig. 4. Working hypothesis of SCA14 pathogenesis by mutant $\gamma \mathrm{PKC}$. Mutant $\gamma \mathrm{PKC}$ is first expressed as a monomer in $\mathrm{PCs}$, followed by oligomerization and aggregation. Oligomerized mutant $\gamma \mathrm{PKC}$ causes aberrant signal transduction in PC dendrites because of its attenuated translocation. This effect results in improper dendritic development and a reduction of dendritic spines. Aggregated mutant $\gamma \mathrm{PKC}$ impairs the UPS and induces ER stress, which leads to the apoptosis of PCs. Both the aberrant morphology of dendrites and apoptosis cause cerebellar dysfunction and contribute to the pathogenesis of SCA14. Although the altered kinase activity of mutant $\gamma \mathrm{PKC}$ might be involved in these dysfunctions, the mechanism remains unclear. 
$\gamma \mathrm{PKC}$ by stabilizing its structure. Moreover, the high basal kinase activity of mutant $\gamma \mathrm{PKC}$ might be related to its aggregate-prone property (24). Further studies are necessary to elucidate how the altered kinase activity of mutant $\gamma \mathrm{PKC}$ affects the functioning of cerebellar PCs.

The mechanism of mutant $\gamma \mathrm{PKC}$ that causes PC dysfunction is partly common to various neurodegenerative diseases $(16,21)$. Therefore, compounds that are effective for the treatment of SCA14 could be available for the treatment of other neurodegenerative disorders. Because mutant $\gamma$ PKC-GFP rapidly induced aggregation and apoptosis within a few days in SH-SY5Y cells (31), this screening system could be beneficial for the exploration of novel candidates for therapeutics against SCA14, and these candidates might be effective for other neurodegenerative diseases.

\section{References}

1 Paulson HL. The spinocerebellar ataxias. J Neuroophthalmol. 2009;29:227-237.

2 Durr A. Autosomal dominant cerebellar ataxias: polyglutamine expansions and beyond. Lancet Neurol. 2010;9:885-894.

3 Williams AJ, Paulson HL. Polyglutamine neurodegeneration: protein misfolding revisited. Trends Neurosci. 2008;31:521528.

4 Chen DH, Brkanac Z, Verlinde CL, Tan XJ, Bylenok L, Nochlin $\mathrm{D}$, et al. Missense mutations in the regulatory domain of PKC gamma: a new mechanism for dominant nonepisodic cerebellar ataxia. Am J Hum Genet. 2003;72:839-849.

5 Chen DH, Cimino PJ, Ranum LP, Zoghbi HY, Yabe I, Schut L, et al. The clinical and genetic spectrum of spinocerebellar ataxia 14. Neurology. 2005;64:1258-1260.

6 Hiramoto K, Kawakami H, Inoue K, Seki T, Maruyama H, Morino $\mathrm{H}$, et al. Identification of a new family of spinocerebellar ataxia type 14 in the Japanese spinocerebellar ataxia population by the screening of PRKCG exon 4. Mov Disord. 2006;21: 1355-1360.

7 Nishizuka Y. Protein kinase C and lipid signaling for sustained cellular responses. FASEB J. 1995;9:484-496.

8 Saito N, Shirai Y. Protein kinase C gamma (PKC gamma): function of neuron specific isotype. J Biochem (Tokyo). 2002;132: 683-687.

9 Chen C, Kano M, Abeliovich A, Chen L, Bao S, Kim JJ, et al. Impaired motor coordination correlates with persistent multiple climbing fiber innervation in PKC gamma mutant mice. Cell. 1995;83:1233-1242.

10 Sakai N, Sasaki K, Ikegaki N, Shirai Y, Ono Y, Saito N. Direct visualization of the translocation of the gamma-subspecies of protein kinase $\mathrm{C}$ in living cells using fusion proteins with green fluorescent protein. J Cell Biol. 1997;139:1465-1476.

11 Shirai Y, Sakai N, Saito N. Subspecies-specific targeting mechanism of protein kinase C. Jpn J Pharmacol. 1998;78:411-417.

12 Sakai N, Tsubokawa H, Matsuzaki M, Kajimoto T, Takahashi E, Ren Y, et al. Propagation of gammaPKC translocation along the dendrites of Purkinje cell in gammaPKC-GFP transgenic mice. Genes Cells. 2004;9:945-957.
13 Seki T, Adachi N, Ono Y, Mochizuki H, Hiramoto K, Amano T, et al. Mutant protein kinase Cgamma found in spinocerebellar ataxia type 14 is susceptible to aggregation and causes cell death. J Biol Chem. 2005;280:29096-29106.

14 Seki T, Takahashi H, Adachi N, Abe N, Shimahara T, Saito N, et al. Aggregate formation of mutant protein kinase $\mathrm{C}$ gamma found in spinocerebellar ataxia type 14 impairs ubiquitin-proteasome system and induces endoplasmic reticulum stress. Eur J Neurosci. 2007;26:3126-3140.

15 Seki T, Shimahara T, Yamamoto K, Abe N, Amano T, Adachi N, et al. Mutant gammaPKC found in spinocerebellar ataxia type 14 induces aggregate-independent maldevelopment of dendrites in primary cultured Purkinje cells. Neurobiol Dis. 2009;33:260 273.

16 Ross CA, Poirier MA. Protein aggregation and neurodegenerative disease. Nat Med. 2004;10 Suppl:S10-S17.

17 Ross CA, Pickart CM. The ubiquitin-proteasome pathway in Parkinson's disease and other neurodegenerative diseases. Trends Cell Biol. 2004;14:703-711.

18 Goldberg AL. Protein degradation and protection against misfolded or damaged proteins. Nature. 2003;426:895-899.

19 Grune T, Jung T, Merker K, Davies KJ. Decreased proteolysis caused by protein aggregates, inclusion bodies, plaques, lipofuscin, ceroid, and 'aggresomes' during oxidative stress, aging, and disease. Int J Biochem Cell Biol. 2004;36:2519-2530.

20 Yamamoto K, Seki T, Adachi N, Takahashi T, Tanaka S, Hide I, et al. Mutant protein kinase $\mathrm{C}$ gamma that causes spinocerebellar ataxia type 14 (SCA14) is selectively degraded by autophagy. Genes Cells. 2010;15:425-438.

21 Glabe CG. Common mechanisms of amyloid oligomer pathogenesis in degenerative disease. Neurobiol Aging. 2006;27: $570-575$.

22 Adachi N, Kobayashi T, Takahashi H, Kawasaki T, Shirai Y, Ueyama T, et al. Enzymological analysis of mutant protein kinase $\mathrm{C}$ \{gamma\} causing spinocerebellar ataxia type 14 and dysfunction in $\mathrm{Ca} 2+$ homeostasis. J Biol Chem. 2008;283:1985419863.

23 Verbeek DS, Knight MA, Harmison GG, Fischbeck KH, Howell BW. Protein kinase $\mathrm{C}$ gamma mutations in spinocerebellar ataxia 14 increase kinase activity and alter membrane targeting. Brain. 2005;128:436-442.

24 Asai H, Hirano M, Shimada K, Kiriyama T, Furiya Y, Ikeda M, et al. Protein kinase $\mathrm{C}$ gamma, a protein causative for dominant ataxia, negatively regulates nuclear import of recessive-ataxiarelated aprataxin. Hum Mol Genet. 2009;18:3533-3543.

25 Hofmann J. The potential for isoenzyme-selective modulation of protein kinase C. FASEB J. 1997;11:649-669.

26 Verbeek DS, Goedhart J, Bruinsma L, Sinke RJ, Reits EA. $\mathrm{PKC}$ \{gamma\} mutations in spinocerebellar ataxia type 14 affect $\mathrm{C} 1$ domain accessibility and kinase activity leading to aberrant MAPK signaling. J Cell Sci. 2008;121:2339-2349.

27 Hirano M, Yamamoto A, Mori T, Lan L, Iwamoto TA, Aoki M, et al. DNA single-strand break repair is impaired in aprataxinrelated ataxia. Ann Neurol. 2007;61:162-174.

28 Schrenk K, Kapfhammer JP, Metzger F. Altered dendritic development of cerebellar Purkinje cells in slice cultures from protein kinase Cgamma-deficient mice. Neuroscience. 2002;110:675689.

29 Crowe JH. Trehalose as a "chemical chaperone": fact and fantasy. Adv Exp Med Biol. 2007;594:143-158. 
30 Tanaka M, Machida Y, Niu S, Ikeda T, Jana NR, Doi H, et al. Trehalose alleviates polyglutamine-mediated pathology in a mouse model of Huntington disease. Nat Med. 2004;10: 148-154.

31 Seki T, Abe-Seki N, Kikawada T, Takahashi H, Yamamoto K, Adachi N, et al. Effect of trehalose on the properties of mutant \{gamma\}PKC, which causes spinocerebellar ataxia type 14, in neuronal cell lines and cultured Purkinje cells. J Biol Chem. 2010;285:33252-33264.
32 Seki T, Takahashi H, Yamamoto K, Ogawa K, Onji T, Adachi N, et al. Congo red, an amyloid-inhibiting compound, alleviates various types of cellular dysfunction triggered by mutant protein kinase $\mathrm{C} \gamma$ that causes spinocerebellar ataxia type 14 (SCA14) by inhibiting oligomerization and aggregation. J Pharmacol Sci. 2010;114:206-216.

33 Frid P, Anisimov SV, Popovic N. Congo red and protein aggregation in neurodegenerative diseases. Brain Res Rev. 2007;53: 135-160. 